\title{
The Role of the Mean State in Meridional Mode Structure and Growth ${ }^{\mathscr{O}}$
}

\author{
CRistian MARTINEZ-VILlalobos AND DANiEl J. VimONT \\ Atmospheric and Oceanic Sciences Department, University of Wisconsin-Madison, Madison, Wisconsin
}

(Manuscript received 3 August 2015, in final form 3 February 2016)

\begin{abstract}
This study uses a simple linear coupled model to investigate the role of the WES feedback and ITCZ mean states in meridional mode variability. Optimal structures that maximize transient growth are calculated for mean states characteristic of boreal spring and boreal fall in the tropical Atlantic. During boreal spring the leading optimal structure is a zonal mode that propagates westward and does not resemble the observed meridional mode. In contrast, the leading optimal structure during fall is a sea surface temperature (SST) monopole over the Northern Hemisphere (NH) that propagates equatorward and westward and that closely matches meridional mode variability during this season. It is found that the boreal fall optimal growth greatly exceeds growth of the corresponding optimal during boreal spring, despite the observed boreal spring peak in Atlantic meridional mode variance.

Sensitivity studies are used to explore the role of Northern or Southern Hemisphere initial conditions, ITCZ width, and ITCZ location in meridional mode growth and structure. It is found that growth is favored (i) for optimal structures that originate in the Northern Hemisphere, especially for boreal fall mean states; (ii) for symmetric mean states, equatorially symmetric structures maximize growth under narrow ITCZ configurations, and antisymmetric structures maximize growth under wider ITCZ configurations; and (iii) for antisymmetric mean states (and realistic ITCZ width), growth is maximized when the ITCZ is located off of the equator. The implications of these findings are discussed.
\end{abstract}

\section{Introduction}

Meridional modes of climate variability are the dominant source of ocean and atmosphere variability in the tropical Atlantic [the Atlantic meridional mode (AMM); Moura and Shukla 1981; Chang et al. 1997; Servain et al. 1999; Chiang and Vimont 2004] and play a secondary role to El Niño-Southern Oscillation (ENSO) as a source of ocean and atmosphere variability in the Pacific [the Pacific meridional mode (PMM); Chiang and Vimont 2004]. Meridional mode variations have also been studied in the South Pacific (Zhang et al. 2014a), the western Pacific (Wang et al. 2012), and the Indian Ocean (Wu et al. 2008). Meridional modes are characterized by an anomalous meridional SST gradient that drives an anomalous interhemispheric boundary

Supplemental information related to this paper is available at the Journals Online website: http://dx.doi.org/10.1175/JCLI-D-15-0542.s1.

Corresponding author address: Cristian Martinez-Villalobos, 1225 W. Dayton St., Madison, WI 53706.

E-mail: cmartinezvil@wisc.edu layer flow from cold to warm waters. Associated with this anomalous boundary layer flow is an ITCZ shift in the direction of the anomalously warm hemisphere (Hastenrath and Heller 1977). The ubiquity of meridional mode variations in the tropical climate system motivates further understanding of the role of the mean state in meridional mode structure and variation.

Meridional modes of climate variability emerge as a result of a positive feedback between wind, evaporation, and SST (the WES feedback; Xie and Philander 1994; Chang et al. 1997). The WES feedback has been explained in the following way: An SST anomaly in one hemisphere creates a cross-equatorial, interhemispheric SST and pressure gradient that drives a meridional flow from the cold hemisphere to the warm one. The Coriolis force deflects the meridional flow to the right in the Northern Hemisphere and to the left in the Southern Hemisphere, which would reinforce the mean easterly trades in the cold hemisphere and reduce the mean easterly trades in the warm hemisphere. This change in wind speed modulates the evaporative latent heat flux at the surface, reinforcing the original SST gradient. Vimont (2010) shows that under the quasigeostrophic 
approximation neither the cross equatorial SST gradient nor the reversal in sign of the Coriolis parameter at the equator is necessary for growth via a WES feedback. Instead, a meridional variation in mean absolute vorticity is sufficient to produce instability and westward propagation of meridional mode structures. Additional feedbacks involving vertical mixing in the ocean (Xie and Philander 1994) and cloud radiative effects (Tanimoto and Xie 2002; Xie and Saito 2001; Evan et al. 2013) have been shown to influence meridional mode variation.

The mean state can influence meridional mode variations through the ITCZ structure, the mean trade wind strength and location, and the variation in stochastic forcing that is likely required for meridional mode variations in nature (Xie 1999; Vimont 2010). The mean ITCZ structure can affect how the atmosphere responds to tropical SST variations via either deep heating (Gill 1980; Zebiak 1986; Battisti et al. 1999) or boundary layer convergence and ventilation (Lindzen and Nigam 1987; Battisti et al. 1999). Mean trade winds influence the strength of the WES feedback via the role of wind speed variations on evaporation (Xie and Philander 1994; Czaja et al. 2002; Vimont et al. 2009; Vimont 2010; Zhang et al. 2014b). The WES feedback has also been implicated in explaining the mean ITCZ state asymmetry in the first place (Xie and Philander 1994; Xie and Saito 2001; Takahashi and Battisti 2007). The study of the ITCZ mean state and variability remains an area of active research (Tomas and Webster 1997; Toma and Webster 2010a,b; Frierson et al. 2013; Schneider et al. 2014; Bischoff and Schneider 2014). External and/or stochastic forcing influences meridional mode variance primarily through geographic variations in trade wind variations and associated surface heat flux to the respective subtropical oceans, especially during winter (Nobre and Shukla 1996). ENSO and the North Atlantic Oscillation (NAO) have both been shown to force the AMM (Xie and Tanimoto 1998; Czaja et al. 2002; Chiang and Vimont 2004; Kossin and Vimont 2007; Penland and Hartten 2014), while in the Pacific the North Pacific Oscillation (NPO) (Rogers 1981; Linkin and Nigam 2008) has been shown as a source of external forcing for the PMM (Chiang and Vimont 2004; Chang et al. 2007; Vimont et al. 2009).

Meridional mode variability over the Atlantic possesses a strong seasonality with peak variance in boreal spring. During this season, meridional mode variability corresponds to the so-called Atlantic SST dipole and consists of an interhemispheric antisymmetric SST pattern, although the anticoherence has been questioned (Houghton and Tourre 1992; Enfield et al. 1999). The boreal spring maximum in meridional mode variability appears to result from enhanced atmospheric forcing during the preceding season (Czaja et al. 2002;
Czaja 2004). However, the contribution of the seasonal cycle (ITCZ location and strength) to the seasonality of meridional mode dynamics is not clear. Meridional mode variability has been less extensively studied during boreal summer and fall when, in the Atlantic, the AMM structure consists mostly of a single SST "monopole" (in contrast to the more familiar equatorially antisymmetric dipole) over the Northern Hemisphere subtropics, with little activity over the Southern Hemisphere (Smirnov and Vimont 2011). The relative roles of internal dynamics and external forcing are also not clear during this season, although compared to spring, external atmospheric forcing is not at its peak.

This study investigates the role of mean state asymmetry on meridional mode structure and growth, including both the effect of asymmetry on internal dynamics and on external forcing. This paper extends the scope of Vimont (2010), who investigates transient growth of meridional mode disturbances under an equatorially symmetric mean state. The remainder of this paper is organized as follows. Section 2 will describe the model and methods utilized. Section 3 will present the optimal results under symmetric and asymmetric ITCZ states and some sensitivity tests. Finally section 4 will present the conclusions.

\section{Model and analysis}

\section{a. Model}

We use a linear dynamical model to investigate growth of disturbances that resemble meridional modes in the tropics. The model we use is the same as that used by Vimont (2010) and consists of a Gill-Matsuno model of the atmosphere coupled with a thermodynamic "slab" ocean model:

$$
\frac{\partial}{\partial t}\left(\begin{array}{l}
u \\
v \\
\phi \\
T
\end{array}\right)=\left[\begin{array}{cccc}
-\varepsilon & \beta y & -\frac{\partial}{\partial x} & 0 \\
-\beta y & -\varepsilon & -\frac{\partial}{\partial y} & 0 \\
-c^{2} \frac{\partial}{\partial x} & -c^{2} \frac{\partial}{\partial y} & -\varepsilon & -K_{q}(y) \\
\alpha(y) & 0 & 0 & -\varepsilon_{T}+\gamma \nabla^{2}
\end{array}\right]\left(\begin{array}{l}
u \\
v \\
\phi \\
T
\end{array}\right) .
$$

The model and constants are described fully in Vimont (2010) and Table 1. Note that the ocean is coupled to the atmosphere via the WES parameter $\alpha(y)[\alpha(y)=$ $\left.\hat{\alpha}(y) /\left(\rho_{o} c_{o} H_{o}\right)\right]$ and that the atmosphere is coupled to the ocean via deep heating, which is linearly proportional to the SST anomaly via the parameter $K_{q}(y)$. 
TABLE 1. Model parameters.

\begin{tabular}{cl}
\hline \hline Parameters & \multicolumn{1}{c}{ Value } \\
\hline$\rho_{o}$ & $10^{3} \mathrm{~kg} \mathrm{~m}^{-3}$ \\
$c_{o}$ & $4.2 \times 10^{3} \mathrm{~J} \mathrm{~kg}^{-1} \mathrm{~K}^{-1}$ \\
$H_{o}$ & $40 \mathrm{~m}$ \\
$\varepsilon$ & $2 \mathrm{day}^{-1}$ \\
$\varepsilon_{T}$ & $120 \mathrm{day}^{-1}$ \\
$\gamma$ & $10^{3} \mathrm{~m}^{2} \mathrm{~s}^{-1}$ \\
\hline
\end{tabular}

The nondimensionalized (Vimont 2010) version of (1) forms our coupled model, which is spectrally decomposed into a single zonal harmonic $\left(2 \pi / k=120^{\circ}\right)$ and discretized in the meridional direction using centered differencing. A sponge layer of enhanced linear damping is applied to the meridional boundaries to eliminate spurious boundary waves. Additional details of the model can be found in Vimont (2010). Note that this model does not include other physical processes that may influence tropical atmosphereITCZ-SST interactions such as inertial instability (Tomas and Webster 1997; Toma and Webster 2010a,b) or realistic Hadley cell variations (Frierson et al. 2013).

\section{b. Background states}

The background mean state affects the model (1) through the location and intensity of the intertropical convergence zone (ITCZ), which affects the meridional structure of $K_{q}(y)$, and the surface zonal winds, which affect the structure and amplitude of the WES parameter $\alpha(y)$. The structure of these two parameters determines coupled system growth, which is a function of the effective coupling $K_{q}(y) \alpha(y)$, everything else held constant (Hirst 1986; appendix A). Here, we find that variations in $K_{q}$ have a larger impact than variations in the WES parameter and hence may play an important role in the structure and evolution of meridional modes.

\section{1) ThE $K_{q}$ AND ITCZ MEAN STATES}

In our model the atmosphere is coupled to the ocean via heating that is proportional to the local SST anomaly. Following Battisti et al. (1999) we parameterize heating to be proportional to the SST anomaly only in regions of mean low-level moisture convergence. A good proxy for mean moisture convergence in the boundary layer is given by $P-E$ :

$$
-\int_{0}^{h} \nabla \cdot\left(\rho_{\mathrm{wv}} \mathbf{u}\right) d z \approx P-E,
$$

where $h$ is the boundary layer top height and $\rho_{\mathrm{wv}}$ is water vapor density. A zonal average over the tropical Atlantic of $P-E$ is used to determine regions of mean moisture convergence and provides a meridional structure for $K_{q}(y)$. Figure 1 shows the location and strength of the
ITCZ (parameterized by $K_{q}$ ) at two different times of the year for the tropical Atlantic. Figure $1 \mathrm{~b}$ represents the most symmetric ITCZ state ( $K_{q}$ in Fig. 1c), which is constrained in shape by the average of $P-E$ in FebruaryApril (FMA). Figure 1e represents the most antisymmetric ITCZ mean state ( $K_{q}$ in Fig. 1f), which is constrained in shape by the average of $P-E$ in August-October (ASO).

\section{2) THE WES PARAMETER $\alpha(y)$ AND SURFACE WINDS}

The WES parameter $\alpha(y)$ is diagnosed by calculating the linearized change in latent heat flux per unit of zonal wind change (Czaja et al. 2002; Vimont 2010). Under the bulk approximation for surface latent heat flux, the WES parameter can be written in terms of the mean latent heat flux LH, the mean zonal wind $\bar{u}$, and the mean wind speed $\bar{w}$ :

$$
-\alpha(x, y) \equiv \mathrm{LH} \frac{\bar{u}}{\bar{w}^{2}} .
$$

The meridional structures for the WES feedback parameter are shown in Fig. 2 for the 3-month averages that correspond to the ITCZ structure in Fig. 1. The solid curve shows the zonal average of the WES feedback for FebruaryApril, and the dashed curve shows the analogous term for August-October conditions over the tropical Atlantic. Note that the seasonal variation of the WES parameter is much smaller than that of the mean heating (Fig. 1).

\section{c. Transient growth analysis}

In the present manuscript, we focus on transient growth of the coupled model rather than growth of individual modes. While Farrell and Ioannou (1996) provide a more complete distinction between the two processes, we provide a brief explanation here. In short, modal growth (growth of a specific eigenvector of the system) is most appropriate for normal systems (which have orthogonal modes), or as $t \rightarrow \infty$. However, in general (and in the case considered herein) the model dynamics are nonnormal, which means that the eigenvectors are not orthogonal. In this case, even if all eigenvectors are damped, growth of particular structures can still occur over a finite time period because of constructive interference between the eigenvectors. In that case, continual excitation of the system by stochastic forcing is required to maintain variance. This paradigm appears to be more appropriate for meridional mode behavior in models and in nature (see the discussion in the introduction). We summarize the distinction here.

The coupled model (1) can be written as $\partial \mathbf{\Phi} / \partial t=\mathbf{M} \boldsymbol{\Phi}$, where $\boldsymbol{\Phi}$ is the state vector $(u, v, \phi, T)$ and the matrix $\mathbf{M}$ is the dynamical system matrix. We can spectrally decompose $\mathbf{M}$ by assuming a modal solution of the form 
a. Feb-Mar-Apr Mean

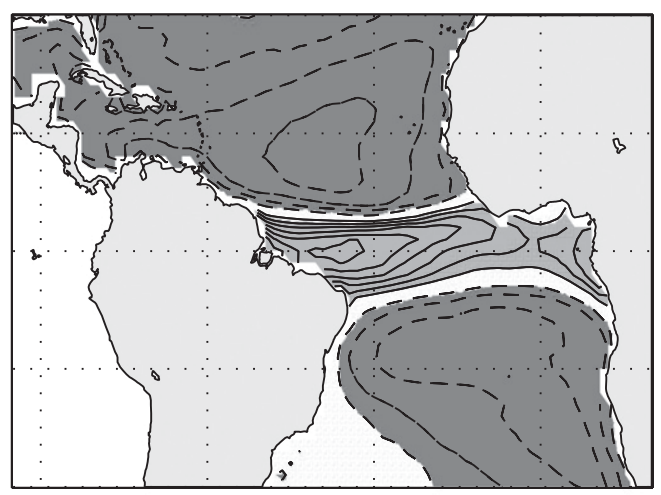

d. Aug-Sep-Oct Mean

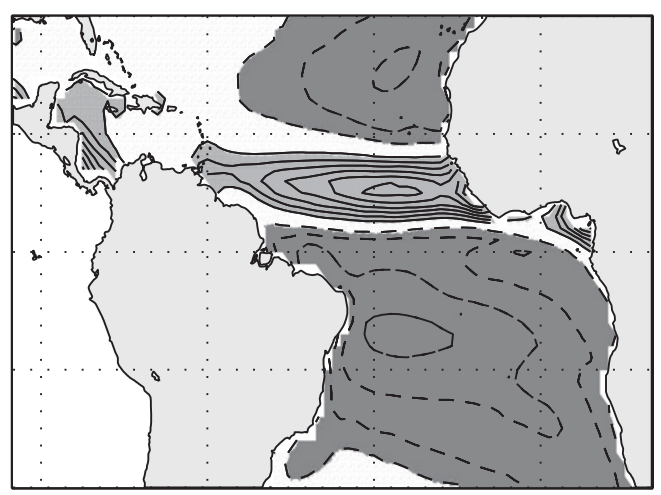

b. Zonal ave

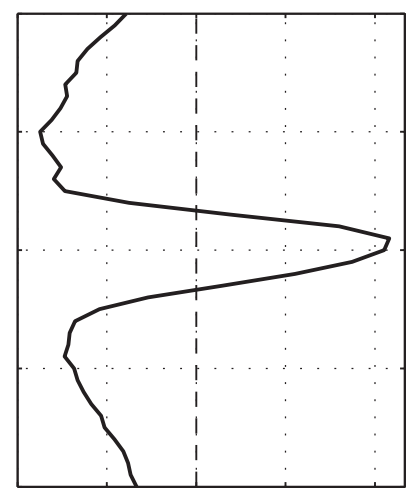

e.

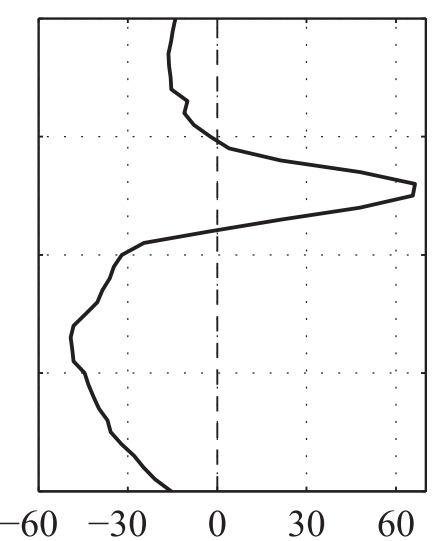

c. $K_{q}$

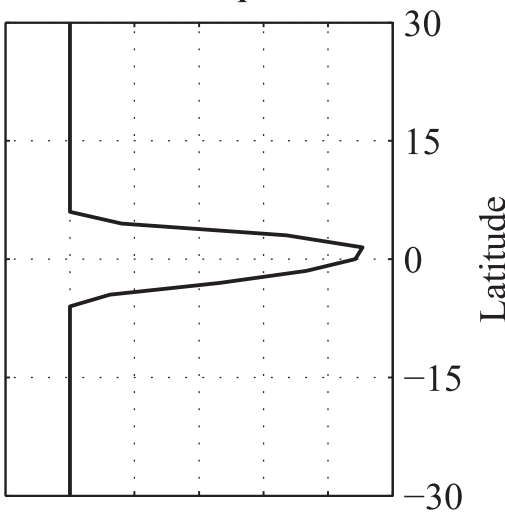

f.

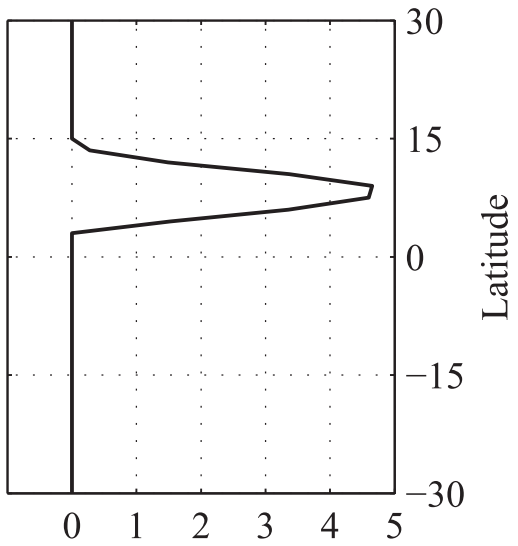

FIG. 1. (a) FMA average of $P-E$ over the Atlantic Ocean for $1981-2010$ (contours; $1.5 \times 10^{-6} \mathrm{~kg} \mathrm{~m}^{-2} \mathrm{~s}^{-1}$ ), (b) zonal average of (a) $\left(10^{-6} \mathrm{~kg} \mathrm{~m}^{-2} \mathrm{~s}^{-1}\right)$, and (c) $K_{q}(y)\left(10^{-3} \mathrm{~m}^{2} \mathrm{~s}^{-3} \mathrm{~K}^{-1}\right)$. (d)-(f) As in (a)-(c), but for ASO. Monthly 1981-2010 precipitation and latent heat data from ERA-Interim (Dee et al. 2011) were used.

$\Phi_{j}(x, y, t)=\hat{\Phi}_{j}(y) \exp \left[i\left(k x-\omega_{j} t\right)\right]$, in which case we obtain the following eigenvalue problem:

$$
\mathbf{M} \Phi_{j}=-i \omega_{j} \Phi_{j} .
$$

The resulting eigenfunctions of $\mathbf{M}$ are the "modes" of the system. For normal systems (in which case all eigenvectors are orthogonal), or as $t \rightarrow \infty$, growth is dominated by the least stable eigenvector of the system (Farrell and Ioannou 1996). However, in our case $\mathbf{M}$ is nonnormal, and hence the modes are not orthogonal. This implies that growth can occur over a finite time period even if all eigenvectors are damped, because of the constructive interference of the eigenvectors.

The nonnormality of $\mathbf{M}$ implies that transient growth may occur over a finite time period even if all of the individual modes are damped. Transient growth is diagnosed by solving (1):

$$
\boldsymbol{\Phi}\left(t-t_{0}\right)=\exp \left[\mathbf{M}\left(t-t_{0}\right)\right] \boldsymbol{\Phi}\left(t_{0}\right) \equiv \mathbf{G}_{\tau} \boldsymbol{\Phi}\left(t_{0}\right),
$$

where $\mathbf{G}_{\tau}$ is the Green's function, which is a function of lag $\tau=t-t_{0}$. Growth over time $\tau$ is measured by the following:

$$
\lambda_{\tau}=\frac{\left|\boldsymbol{\Phi}\left(t-t_{0}\right)\right|_{N}^{2}}{\left|\boldsymbol{\Phi}\left(t_{0}\right)\right|_{L}^{2}}=\frac{\boldsymbol{\Phi}^{\dagger}(\tau) \mathbf{N} \boldsymbol{\Phi}(\tau)}{\boldsymbol{\Phi}^{\dagger}(0) \mathbf{L} \boldsymbol{\Phi}(0)}=\frac{\boldsymbol{\Phi}^{\dagger}(0) \mathbf{G}_{\tau}^{\dagger} \mathbf{N} \mathbf{G}_{\tau} \boldsymbol{\Phi}(0)}{\boldsymbol{\Phi}^{\dagger}(0) \mathbf{L} \boldsymbol{\Phi}(0)}
$$

where subscripts $N$ and $L$ indicate that the norm is taken under a specified final and initial norm kernel, respectively, and $\mathbf{N}$ and $\mathbf{L}$ are the specified final and initial norm kernels in the discretized model. Without loss of generality we can maximize growth of the numerator in (6) under the constraint that the initial condition have length 1 under its respective initial norm. The Lagrangian function $\mathscr{B}$ condenses that information:

$$
\mathscr{B}(\boldsymbol{\Phi}, \lambda)=\boldsymbol{\Phi}^{\dagger}(0) \mathbf{G}_{\tau}^{\dagger} \mathbf{N G} \mathbf{\Phi}_{\tau}(0)-\lambda_{\tau}\left[\boldsymbol{\Phi}^{\dagger}(0) \mathbf{L} \boldsymbol{\Phi}(0)-1\right]
$$




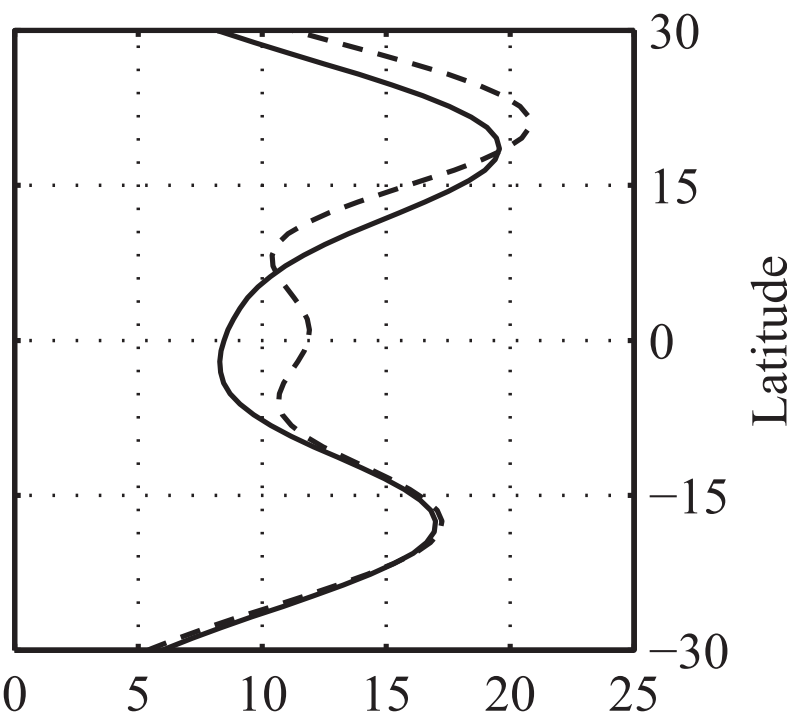

FIG. 2. Zonal mean of the WES parameter $\left(\mathrm{J} \mathrm{m}^{-3}\right)$ over the Atlantic for 1981-2010: FMA average (solid) and ASO average (dashed). Monthly 1981-2010 latent heat and wind data from ERA-Interim (Dee et al. 2011) were used.

By taking the derivative of (7) with respect to elements of the state vector $\boldsymbol{\Phi ( 0 )}$ and setting the result equal to zero we obtain the generalized eigenvalue equation:

$$
\mathbf{G}_{\tau}^{\dagger} \mathbf{N} \mathbf{G}_{\tau} \mathbf{p}_{\tau}=\lambda_{\tau} \mathbf{L}_{\tau} .
$$

Here $\mathbf{p}_{\tau}$ are the initial structures that optimize growth over a time $\tau, \lambda_{\tau}$ is the growth under the specified final and initial norms, and the symbol $\dagger$ denotes Hermitian conjugate. The evolved optimal structures at time $\tau$ can be found by simply calculating

$$
\mathbf{q}(\tau)=\mathbf{G}_{\tau} \mathbf{p}_{\tau} .
$$

We will refer to the structures represented by $\mathbf{p}_{\tau}$ as optimal initial structures, or simply "optimals," and structures in $\mathbf{q}(\tau)$ as the optimal final structures.

Equation (6) indicates that growth is norm-dependent. The nondimensionalization of (1) however, results in an arbitrary norm because in our case, the choice of a reference SST for nondimensionalization is arbitrary. In practice, we are interested in coupled structures with time scales that are long compared to the atmospheric damping time scale ( 2 days). For this reason we can consider only the growth of SST because for time scales of interest the atmosphere will effectively be in equilibrium with the SST. To evaluate our choice of nondimensional parameters and to provide an illustration of the use of a final norm, we reevaluate transient growth under the equatorially symmetric case considered by Vimont (2010). We compare results calculated under a specified final "SST norm" $\mathbf{N}_{\mathrm{SST}}=\operatorname{diag}(\varepsilon, \varepsilon, \varepsilon, 1)$, where $\varepsilon \ll 1$, with results in Vimont (2010) that were calculated under a Euclidean final norm (in the nondimensionalized system) in which $\mathbf{N}=\mathbf{1}$, the identity matrix. Here, we use $\varepsilon=10^{-9}$ rather than $\varepsilon=0$ for numerical stability. Our construction of the SST norm emphasizes growth of SST variance.

Figure 3 left column shows the initial and final 140-day optimals using $\mathbf{N}_{\mathrm{SST}}$. Comparing to Fig. 7 in Vimont (2010) (135-day optimal) we can see that the structures are essentially the same. Also the growth rates (Fig. 6 in Vimont 2010) of the first three optimals are, at a good precision, unchanged (not shown). Figure $3 e$ shows the Euclidean growth for the 140-day Euclidean and SST optimals. Both growth curves are nearly identical. This is because our nondimensionalization gives much more relative weight to growth of SST anomalies than of atmospheric fields. In the remainder of the paper we will simply use $\mathbf{N}=\mathbf{1}$, the identity matrix.

To illustrate the effect of varying initial norms, we calculate optimal initial and final conditions under a "Northern Hemisphere norm" in which initial conditions are constrained to be north of the equator. This is achieved by setting $\mathbf{L}_{\mathrm{NH}}=\operatorname{diag}[\varepsilon H(y)+H(-y)]$, where $H(y)$ is the Heaviside function, and again $\varepsilon \ll 1$ and is not equal to 0 for numerical stability. This norm kernel penalizes an initial condition in the Southern Hemisphere and effectively allows unconstrained initial conditions in the Northern Hemisphere. ${ }^{1}$ Optimal initial and final conditions calculated under the Northern Hemisphere initial norm and Euclidean final norm are shown in Fig. 3 (right). Results show an initial condition with amplitude in the Northern Hemisphere only (see appendix B). The response in this case is qualitatively different than that of the Euclidean initial norm in Fig. 3. In particular, the response is asymmetric in that it includes both equatorially symmetric and antisymmetric (e.g., a dipole) structures in SST. This is expected given the asymmetric initial condition.

\section{Structure and growth under asymmetric mean states}

In this section we calculate growth and evolution of optimal structures under various mean state geometries. In section 3a, we analyze growth under "observed" mean states for boreal spring and fall and find that a

\footnotetext{
${ }^{1}$ For the same argument, an SST initial norm does not make physical sense for long lead times, as it would penalize initial SST anomalies and allow unconstrained atmospheric initial anomalies. For long lead times, these atmospheric anomalies are simply the structures that most effectively generate SST anomalies that ultimately are responsible for the long-term growth.
} 
a) 140 days Initial Optimal

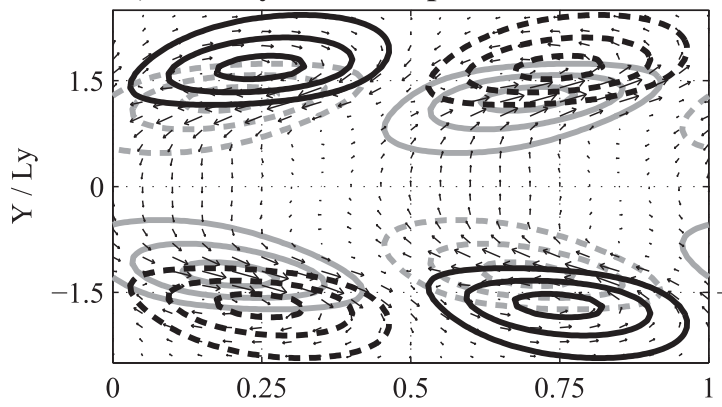

b) 140 days Final Optimal

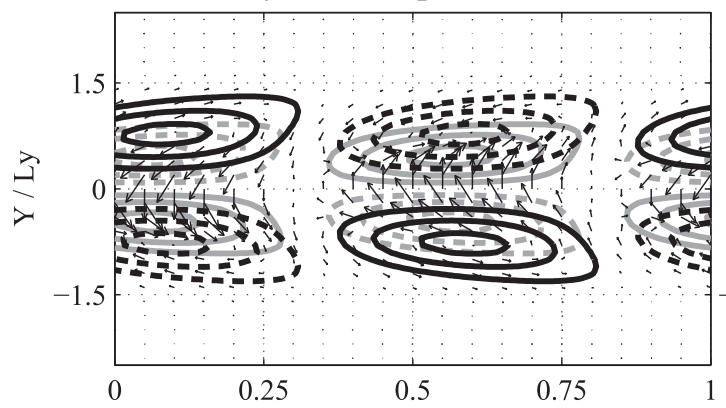

c) 100 days Initial Optimal

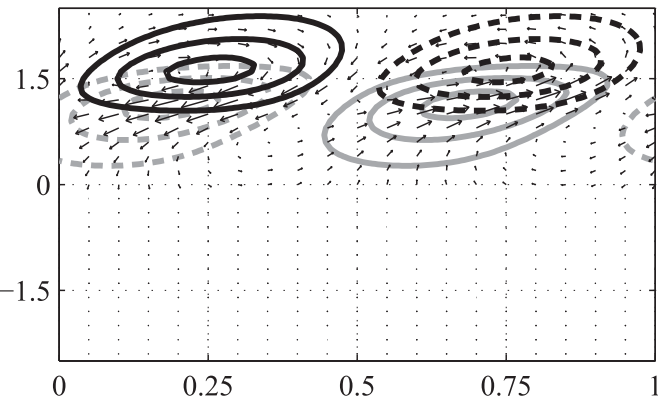

d) 100 days Final Optimal

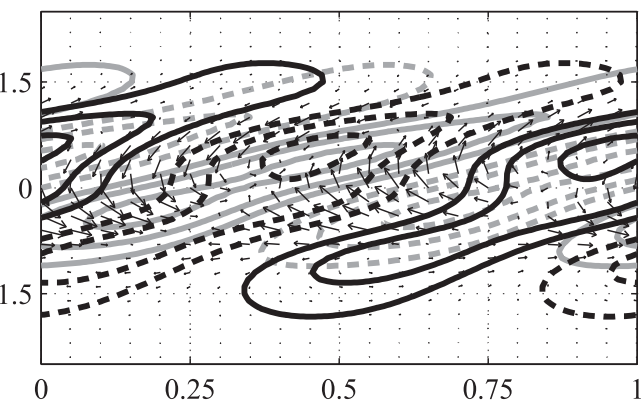

e) Growth

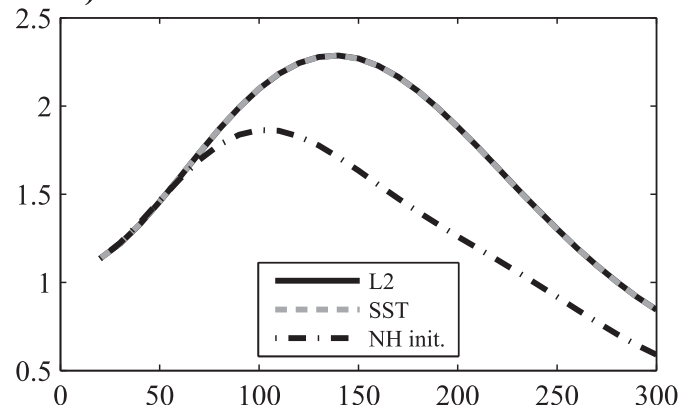

FIG. 3. Optimal (a),(c) initial and (b),(d) final structures calculated under the same mean state as used in Vimont (2010) for 140 days under the full (unconstrained) initial norm kernel and SST final norm kernel in (a), (b) and for 100 days under the NH initial norm kernel with full (L2) final norm kernel in (c),(d). SST is represented by gray contours, low-level geopotential height by black contours, and low-level wind fields by the arrows. Contours are shown at increments of 0.3 times the maximum value of the field, solid contours denote positive values, and dashed contours negative ones. Units on the $x$ and $y$ axes have been scaled by the zonal wavelength $\left(2 \pi / k=120^{\circ}\right)$ and the equatorial radius of deformation $\left(L_{y} \sim 10^{\circ}\right.$ for the parameters of this model). (e) Growth (under the L2 norm) as a function of days of the optimal calculated under the full L2 norm (Vimont 2010) (black line behind gray dashed line), optimal calculated under the SST norm, shown in (a) and (b) (gray dashed line), and optimal calculated under the NH initial norm, shown in (c) and (d) (black dashed-dotted line).

symmetric (asymmetric) structure produces optimal growth under the spring (fall) conditions. We investigate the role of mean state asymmetry and ITCZ width in section $3 \mathrm{~b}$ and discuss differences in the structures and growth in section $3 \mathrm{c}$.

\section{a. Optimal structures in boreal spring and fall}

In this section we will calculate the optimal structures that maximize transient growth over boreal spring and fall, which are the seasons characterized by extreme mean locations of the ITCZ in the Atlantic. To address the asymmetry between the Northern Hemisphere (NH) and Southern Hemisphere (SH) extratropical forcing on meridional mode variability, we also calculate the optimals and growth rates that arise when we constrain the optimal initial conditions to either the Northern or Southern Hemisphere. The results shown in this section should hold qualitatively well over the eastern Pacific as the mean WES and ITCZ states there are similar to the Atlantic. 
a) 140 days Initial Optimal

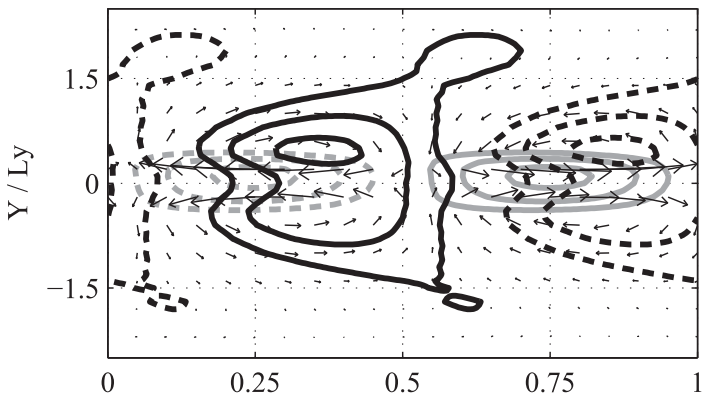

b) 140 days Final Optimal

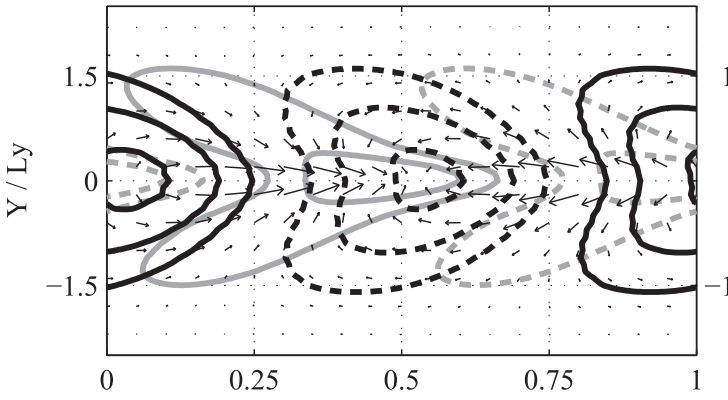

c) 140 days Initial Optimal (NH Constr.)

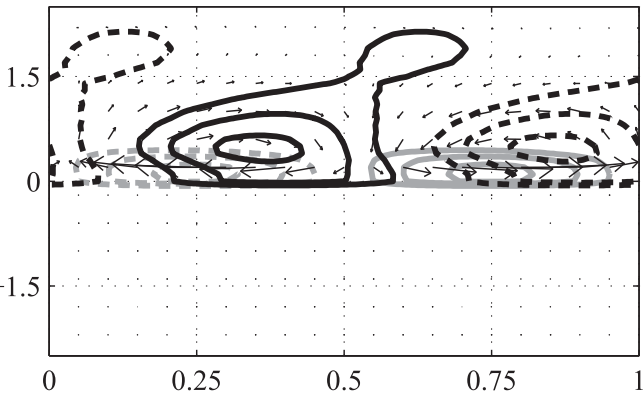

d) 140 days Final Optimal (NH Constr.)

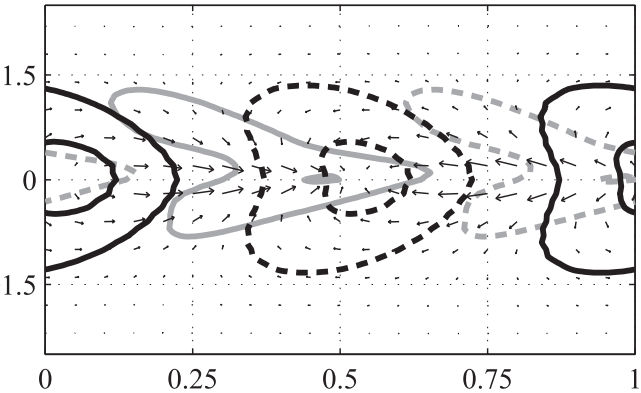

e) Transient growth

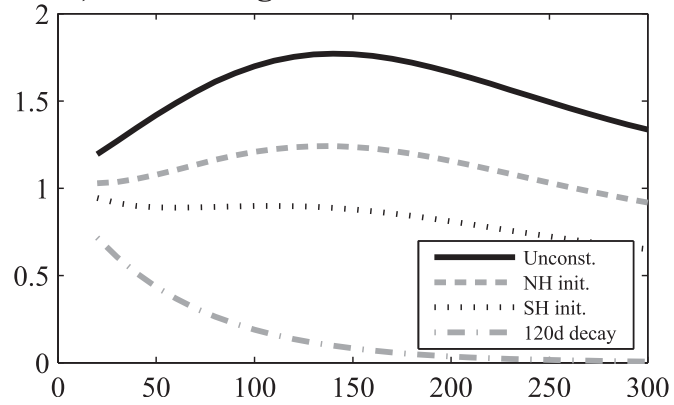

FIG. 4. Optimal (a),(c) initial and (b),(d) final structures calculated under the FMA mean state for 140 days under the full (unconstrained) initial norm kernel in (a),(b) and for 140 days under the NH initial norm kernel in (c),(d). SST is represented by gray contours, low-level geopotential height by black contours, and low-level wind fields by the arrows. Contours are shown at increments of 0.3 times the maximum value of the respective initial condition fields, solid contours denote positive values, and dashed contours negative ones. The lengths of the velocity arrows are drawn relative to the maximum wind speed of the respective initial conditions. Units on the $x$ and $y$ axes have been scaled by the zonal wavelength $\left(2 \pi / k=120^{\circ}\right)$ and the equatorial radius of deformation $\left(L_{y} \sim 10^{\circ}\right.$ for the parameters of this model). (e) Growth $\lambda_{\tau}$ as a function of days for the unconstrained optimal initial condition (black solid line), optimal NH initial condition (gray dashed line), optimal SH initial condition (black dotted line), and a 120-day decay curve (gray dashed-dotted line).

Figure 4 shows the 140-day optimal initial and final structures under boreal spring mean states. Figure $4 \mathrm{a}$ depicts the unconstrained optimal initial condition, and Fig. $4 \mathrm{c}$ shows the case in which the initial condition is constrained to the NH. The unconstrained optimal (Fig. 4a) is nearly symmetric about the equator (it would be perfectly symmetric if both $\alpha$ and $K_{q}$ were exactly symmetric), both at the beginning and during its evolution, and propagates westward. Growth of this structure maximizes at 140 days. The optimal final condition strongly resembles the least stable eigenvector of the system (not shown). We note that the least stable eigenvector of the dynamical system matrix is damped, so any growth must occur through nonnormality. The other eigenvectors of the dynamical matrix M are heavily damped for this mean state, so by 140 days the remaining modes (which contribute to the nonnormal growth prior to 140 days) have effectively decayed and the least stable eigenvector is left to decay at its own rate.

When the initial condition is constrained to the Northern Hemisphere (Fig. 4c) the optimal is just the northern half of the unconstrained initial condition (Fig. 4a; see appendix B). Like the unconstrained optimal, 
the NH-constrained optimal grows into a structure (Figs. 4b,d) that strongly resembles the least stable eigenvector of the system in 140 days. The SH-constrained initial condition (not shown) is very similar in structure to the mirror image of the $\mathrm{NH}$-constrained initial condition, although the $\mathrm{NH}$ one grows more than its $\mathrm{SH}$ counterpart (Fig. 4e). The WES feedback is stronger in the Northern Hemisphere (Fig. 2) and the ITCZ is centered slightly north of the equator (Fig. 1c) on average over FMA, which results in a stronger effective coupling over the Northern Hemisphere tropics and a larger growth rate for the NH-constrained initial condition. The SH-constrained optimal decays at a slower rate than the 120 day $^{-1}$ linear oceanic damping rate, implying a positive role of the WES feedback in enhancing SST variance.

The leading optimal does not resemble the antisymmetric AMM structure that dominates variability in the tropical Atlantic during boreal spring. However, we include a discussion of this structure as similar modes do emerge in other coupled models (Noguchi 1998; Xie et al. 1999). We speculate that this optimal could be related to a thermally coupled Walker mode that is observed in more complex models when the atmosphere is coupled to a thermodynamic slab ocean via moisture and heat fluxes (Clement et al. 2011). This optimal also looks similar to one of the unstable modes found in Hirst (1986, their Fig. 9b) when the SST equation includes mean zonal SST advection by an anomalous zonal current but no thermocline feedbacks. In that case, the mean zonal advection produces an effect similar to our WES feedback, leading to similar structure and propagation characteristics.

Over boreal fall the mean state (Figs. 1 and 2) and resulting optimal structures (Fig. 5) are quite different. The unconstrained leading optimal is asymmetric (i.e., a combination of a symmetric and antisymmetric function) about the equator, with most of the amplitude in the $\mathrm{NH}$. The initial condition starts almost exclusively in the $\mathrm{NH}$ and evolves westward and equatorward over time, becoming more symmetric in the process (Figs. 5a,b). This type of "nondipole" behavior is in accord with the Atlantic meridional mode variability observed during boreal summer and fall (see Fig. 2 of Smirnov and Vimont 2011). When the initial condition is constrained to the $\mathrm{NH}$, the optimal initial and final conditions (not shown) are virtually identical to the unconstrained optimal, and so is the growth rate (Fig. 5c). Note that for both the unconstrained and constrained optimal, growth rates for the ASO mean state conditions are nearly double that of the FMA unconstrained optimal, despite similar WES parameters and similar width and amplitude of the ITCZ (and hence $K_{q}$ ). The explanation for this behavior is traced to atmospheric wave dynamics and will be addressed in section $3 \mathrm{c}$. a) 110 days Initial Optimal

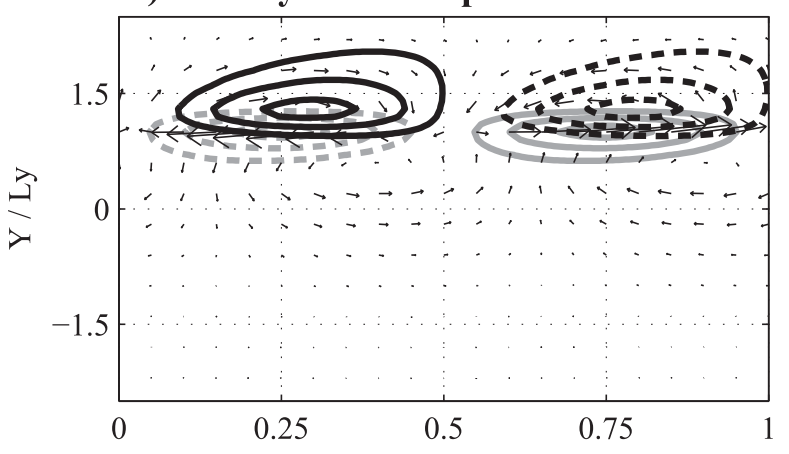

b) 110 days Final Optimal

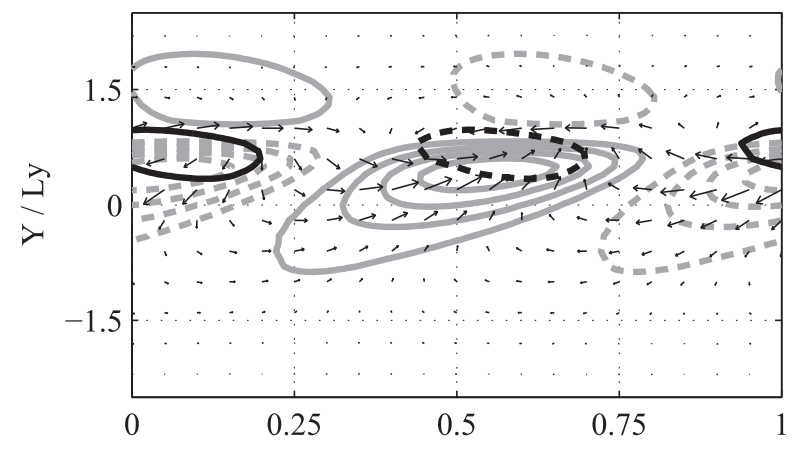

c) Transient growth

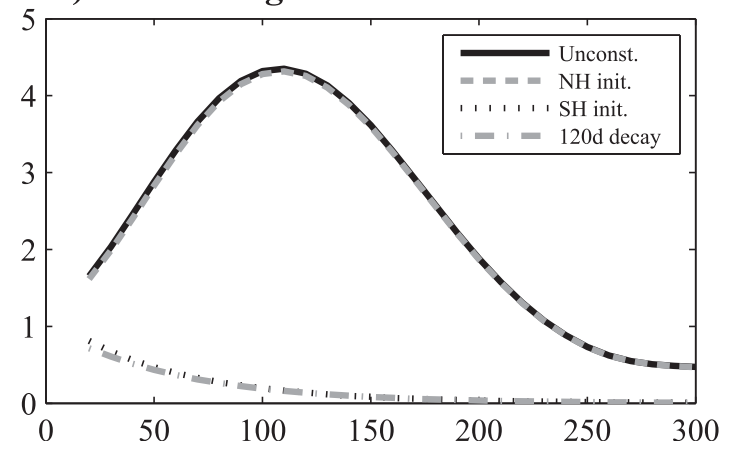

FIG. 5. The 110-day (a) initial and (b) final optimal structures for ASO mean states when the initial condition is unconstrained. SST is represented by gray contours, low-level geopotential height by black contours, and low-level wind fields by the arrows. Contours are shown at increments of 0.3 times the maximum value of the initial condition fields, solid contours denote positive values, and dashed contours negative ones. The lengths of the velocity arrows are drawn relative to the maximum wind speed of the initial condition. Units on the $x$ and $y$ axes have been scaled by the zonal wavelength $\left(2 \pi / k=120^{\circ}\right)$ and the equatorial radius of deformation ( $L_{y} \sim 10^{\circ}$ for the parameters of this model). (c) Growth $\lambda_{\tau}$ as a function of days for the unconstrained optimal initial condition (black solid line; note this is behind the gray dashed line), optimal NH initial condition (gray dashed line), optimal SH initial condition (black dotted line), and a 120-day decay curve (gray dasheddotted line). 
During boreal fall the mean ITCZ is completely contained in the NH tropics, implying that convective support for coupled ocean-atmosphere interaction is asymmetric. Any initial condition in the Southern Hemisphere would quickly die off because the system is uncoupled there (in this model) during this season. We note that the SH behavior is a limitation to our study, as these variations may be coupled through boundary layer adjustment (Lindzen and Nigam 1987; Battisti et al. 1999; Chiang et al. 2001) in nature. Optimals constrained to start in the SH are not well separated and show very little large-scale structure (not shown). Any SH-constrained initial optimal decays at a rate close to the linear oceanic damping rate, as can be seen in Fig. $5 \mathrm{c}$ for the leading SH optimal.

\section{b. The role of ITCZ location and width}

In this section we explore how the mean state affects the growth and symmetry of the optimal response under a symmetric $K_{q}$ mean state. This is motivated by the different response found in Vimont (2010) compared to our result over boreal spring (Fig. 4) and the mismatch between the leading symmetric optimal and the dominant structure of observed ocean-atmosphere variability in the Atlantic. In both cases the mean heating and WES feedback states are (almost) symmetric. Vimont (2010) finds an equatorially antisymmetric optimal response, while our boreal spring response is symmetric. Are there regimes in which the response will be confined to the equator, with no associated interhemispheric boundary layer flow? Will a traditional dipolar antisymmetric optimal response be possible under other circumstances?

To explore these questions we investigate the change in structure and growth of the leading symmetric and antisymmetric optimal responses as a function of the width and location of the coupling regions, in particular the $K_{q}$ parameter. We look at growth rates and initial and final structures for profiles of $K_{q}(y)$ that are parameterized as follows:

$$
K_{q}(y)=\exp \left[-\left(\frac{y-y_{0}}{y_{w}}\right)^{4}\right],
$$

where $y_{0}$ is the central ITCZ location and $y_{w}$ is a characteristic width of the ITCZ. We use this shape since it most closely resembles the profiles constrained by moisture convergence in boreal spring and fall (see Fig. 1). We begin by examining growth for a symmetric ITCZ $\left(y_{0}=0^{\circ}\right)$ with characteristic width of $y_{w}=5^{\circ}, 10^{\circ}, 15^{\circ}$, and $20^{\circ}$ latitude (Fig. 6a). For all cases we use a constant WES feedback parameter of magnitude $13.5 \mathrm{~J} \mathrm{~m}^{-3}$, chosen because it is the average of $\alpha(y)$ in boreal spring between $30^{\circ} \mathrm{S}$ and $30^{\circ} \mathrm{N}$. Optimal structures obtained in this system are fairly insensitive to similar WES feedback structures or amplitudes. The growth rates are somewhat more sensitive, but the hierarchy of growth rates for the different $K_{q}$ profiles remains unchanged, and so do the main conclusions. We note that for all symmetric mean states here the system is linearly stable and the least stable eigenvector of the system is symmetric.

Growth of the leading symmetric and antisymmetric optimal structures under the different coupling regions (Fig. 6a) are shown in Figs. 6b and 6c, respectively. A general increase of the growth is observed for both symmetric and antisymmetric leading optimals as the coupling region gets wider. The rate of increase of the growth as a function of $K_{q}$ width is greater for antisymmetric optimals compared to symmetric ones, indicating that antisymmetric modes preferentially grow via nonnormal processes. This is consistent with results from Xie (1996), who shows that the coupling of the system is proportional to the coupling window width squared for antisymmetric modes. For these model parameters the two narrowest $K_{q}$ configurations produce a symmetric leading optimal, and the two widest $K_{q}$ configurations produce an antisymmetric leading optimal. The importance of the symmetric optimals for a narrow symmetric coupling window is somewhat at odds with the results of Okajima et al. (2003) and highlights the role of the coupling window specification. We have checked that the first optimal remains symmetric under the $5^{\circ} K_{q}$ window for different zonal wavenumbers covering at least the range from $k=0$ to $k=20\left(\pi / 120^{\circ}\right)$ (not shown).

Comparing Fig. 4e to Fig. 5c, it is observed that the leading optimal condition achieves more growth in boreal fall compared to spring. Is this result part of a pattern in which optimal conditions transiently grow more for asymmetric mean states? To investigate this we calculate the transient growth under different $K_{q}(y)$ central locations. We use the $y_{w}=5^{\circ}$ latitude width function (Fig. 6, solid line) but vary $y_{0}$ as $0^{\circ}, 4^{\circ}, 8^{\circ}, 12^{\circ}, 16^{\circ}$, and $20^{\circ}$ latitude for each different case. The same constant WES feedback profile used in the previous section is utilized here. The system is linearly stable under all $K_{q}$ configurations considered. We have restricted this part of the study to optimals with a large antisymmetric component, as they are the ones associated with motions that mostly vary in the north-south axis. For $K_{q}$ centered at $0^{\circ}$ we show the first antisymmetric optimal growth. If we include the symmetric mode, the conclusions derived from this section do not change. Figure 7 shows the transient growth as a function of $\tau$ for the different configurations. There is an increase in the maximum growth of optimals as a function of $y_{0}$, up to a peak that occurs when $y_{0}=13^{\circ}$ latitude $\left(y_{0}=13^{\circ}\right.$ latitude case is not shown). Beyond $y_{0}=13^{\circ}$ latitude the maximum transient growth starts to diminish and starts occurring earlier in the system evolution. 


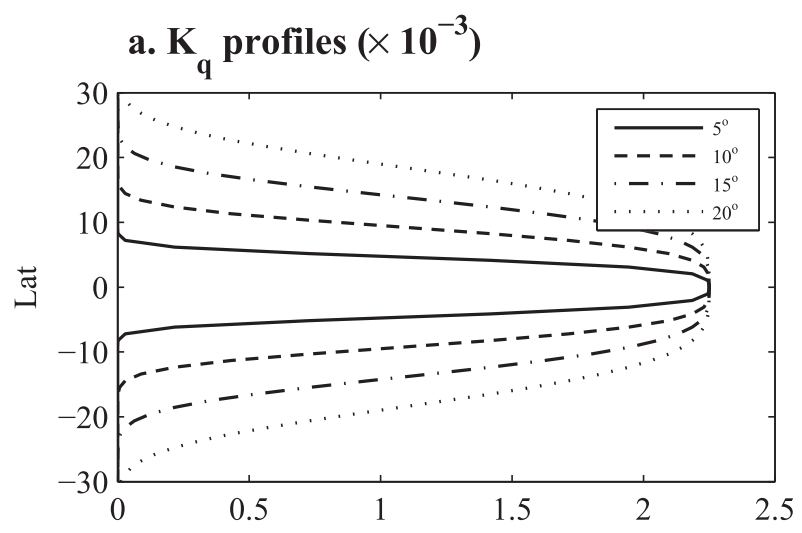

b. Symmetric growth

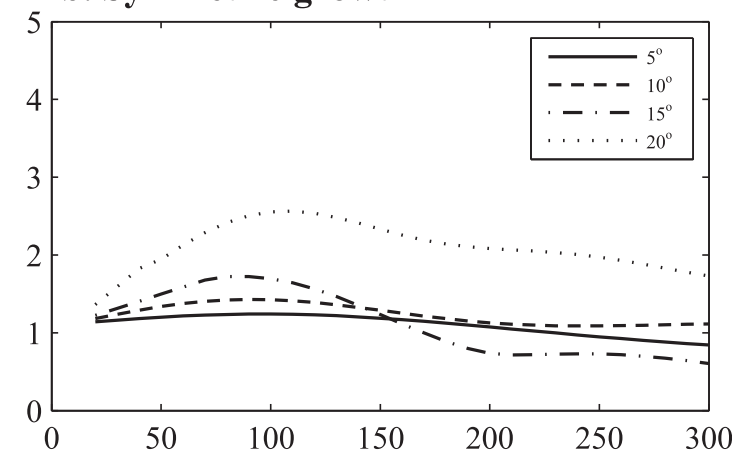

c. Antisymmetric growth

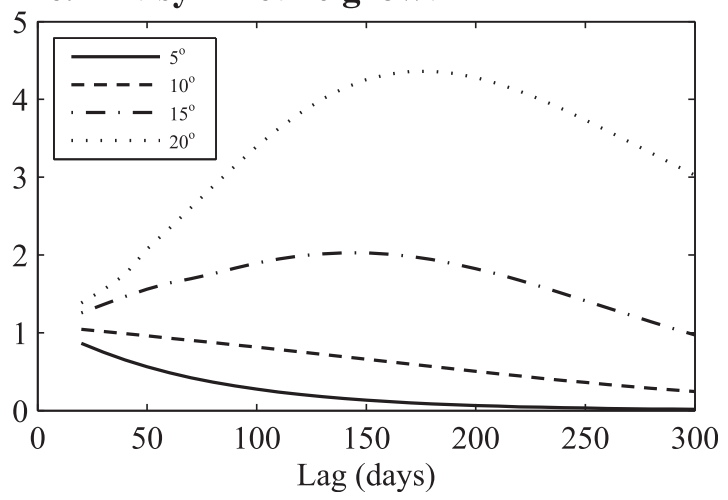

FIG. 6. (a) Different $K_{q}$ curves with a peak of $2.25 \mathrm{~m}^{2} \mathrm{~s}^{-3} \mathrm{~K}^{-1}$. The curves are described in terms of their characteristic width in latitude: $5^{\circ}$ (solid), $10^{\circ}$ (dashed line), $15^{\circ}$ (dashed-dotted line), and $20^{\circ}$ (dotted line). The vertical axis is measured in degrees. (b) Least stable symmetric optimal growth as function of $K_{q}$ characteristic width $y_{w}$. (c) Least stable antisymmetric optimal growth as a function of $K_{q}$ of $y_{w}$.

\section{c. The mean state as a "mode selector"}

The different optimal responses for different symmetric mean states (Fig. 6a) may be understood in terms of the spatial structure of heating of stationary forced atmospheric Rossby and Kelvin waves, which depends on the product $K_{q}(y) T$. In this case, the mean state acts as a mode selector for the atmospheric contribution. To explore the role of ITCZ width and location on the

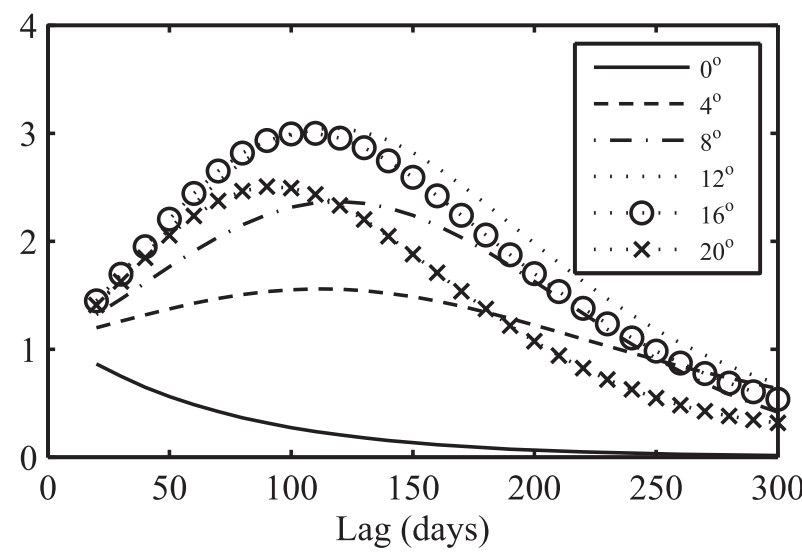

FIG. 7. Growth of the least stable asymmetric mode under mean state given by the shape and magnitude of solid line in Fig. 6a, but centered at $y_{0}=0^{\circ}$ (solid line), $4^{\circ}$ (dashed line), $8^{\circ}$ (dashed-dotted line), $12^{\circ}$ (dotted line), $16^{\circ}$ (circle), and $20^{\circ}$ (cross).

forcing of atmospheric free modes, we calculate the norm of $K_{q}(y) D_{n}(y)$, where $D_{n}(y)$ is the parabolic cylinder function of order $n$ (Gill 1980). This norm roughly indicates the amplitude of excitation of Kelvin and Rossby waves that would be generated by temperature anomalies with structure $D_{n}(y)$ for a given ITCZ structure [note that the norm of $D_{n}(y)=1$ ]. We plot the norm of $K_{q}(y) D_{n}(y)$ for the first three parabolic cylinder functions as a function of $y_{w}$ in Fig. 8a and $y_{0}$ in Fig. 8b.

The transition from the symmetric mode for narrow ITCZ structures to an antisymmetric mode for broader ITCZ structures is examined as a function of ITCZ width (all equatorially centered) in Fig. 8a. When $K_{q}(y)$ is confined to low latitudes, the only region where surface temperature anomalies can excite a significant atmospheric response is confined to the equator. In the case of the $5^{\circ}$ and $10^{\circ}$ latitude width ITCZ cases, only the Kelvin wave and gravest symmetric Rossby mode are effectively excited by surface temperature variations. For wider $K_{q}(y)$ higher-order Rossby modes are activated via the product of $T(y)$ with $K_{q}(y)$, including antisymmetric ones. For reference, the $n=1$ parabolic cylinder function, which is the heating structure that excites the lowest antisymmetric Rossby wave, has a maximum at one Rossby radius away from the equator $(\sqrt{c / \beta})$, or about $10^{\circ}$ of latitude for the parameters used in this study. The symmetric initial condition will excite a damped equatorial Kelvin wave, which interferes with growth in this model through the WES feedback (the Kelvin wave produces easterly anomalies over positive temperature anomalies; see, e.g., Gill 1980). Hence, for wide coupling regions the antisymmetric modes dominate.

Similar arguments explain why the antisymmetric optimal is preferentially excited for ITCZ structures that are centered off the equator. The norm of $D_{n}(y) K_{q}(y)$ is 

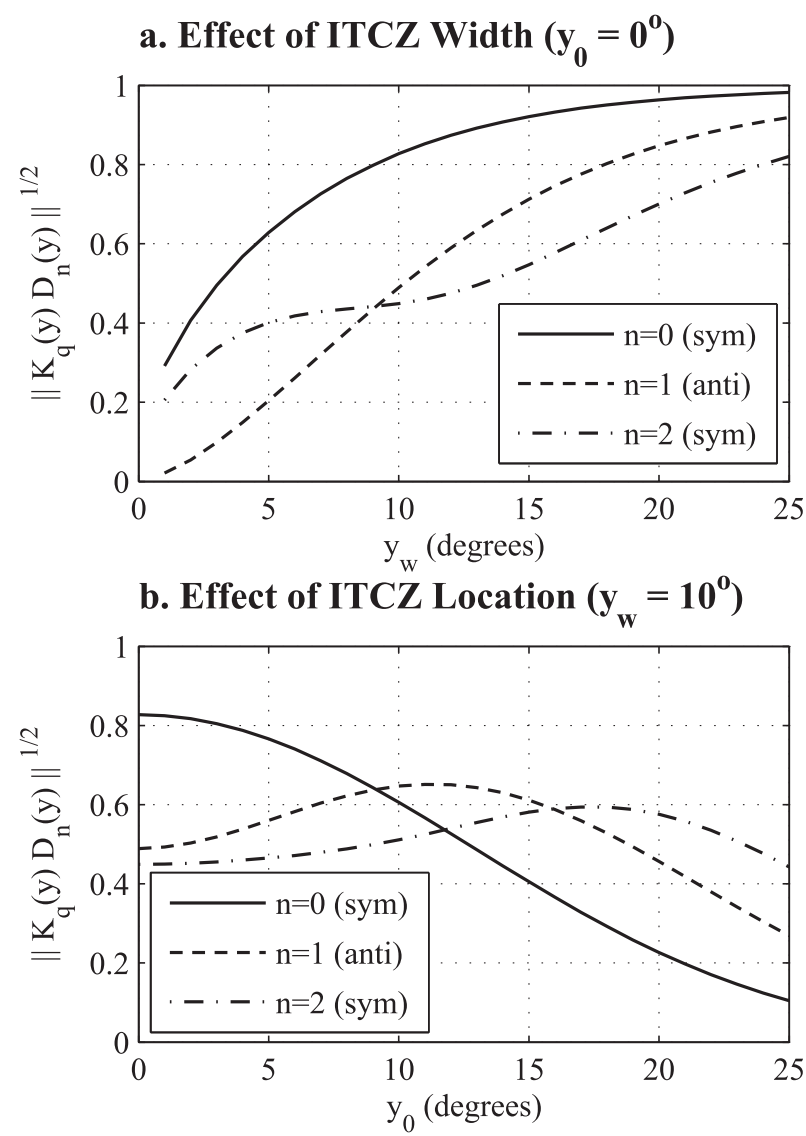

FIG. 8. (a) Norm of $K_{q}(y) D_{n}(y)$ as a function of $y_{w}$ (for $y_{0}=0^{\circ}$ ). (b) Norm of $K_{q}(y) D_{n}(y)$ as a function of $y_{0}$ (for $y_{w}=10^{\circ}$ ). This shows that the effective coupling increases as the ITCZ width increases in (a) and that the ITCZ location acts as a mode selector as it moves poleward in (b).

plotted as a function of ITCZ location in Fig. $8 \mathrm{~b}$ for $y_{w}=5^{\circ}$ latitude. Heating is maximized for the leading symmetric parabolic cylinder function when the ITCZ is symmetric about the equator and decreases as the ITCZ shifts poleward. Still, the damped Kelvin wave response for the leading symmetric parabolic cylinder function reduces growth through the WES feedback. Heating for the second $(n=1$; antisymmetric) parabolic cylinder function maximizes when the ITCZ is centered at about $12^{\circ}$ latitude, near the location of maximum growth in Fig. 6. As the ITCZ moves poleward the effective coupling region from the ocean to the atmosphere moves with it. Vimont (2010) shows that for larger values of the Coriolis parameter the atmospheric geopotential response to heating anomalies becomes more in phase with the surface temperature, which reduces the effectiveness of the WES feedback in generating transient growth. Hence, growth is maximized for antisymmetric structures that originate in the subtropics.

Comparing the growth and structures of the optimal responses under different symmetric coupling regions is not just a theoretical exercise. As previously mentioned, in this work the ocean is coupled to the atmosphere through SST-induced deep heating anomalies. To a first approximation this Gill-like atmospheric model (Gill 1980) can be transformed into a Lindzen and Nigam (1987) (LN)-type model (Neelin 1989) where SST influences the boundary layer pressure gradients that directly drive the boundary layer winds. The equatorial radius of deformation $L_{D}$ is typically smaller in the $\mathrm{LN}$ formulation (Neelin 1989; Vimont 2010). This implies that the same coupling region width (in physical units) will look wider in an LN-type model, further implying different symmetry and growth rates for the leading optimals depending on which kind of model is considered. This is verified using the Vimont (2010) version of an LNtype model, where the convective coupling window affects boundary layer mass ventilation by deep convection. In this case the leading optimal structures remain equatorially antisymmetric for all the coupling windows considered in Fig. 6a (see Fig. S1 of supplementary material).

\section{Conclusions}

The role of the mean state in growth and structure of tropical meridional mode variability was examined using the simple linear coupled model of Vimont (2010). For an equatorial mean state that is largely symmetric (representing boreal spring in the Atlantic) growth is dominated by a zonally propagating eigenmode that does not resemble observed meridional mode variations. Transient growth of meridional-mode-like structures is larger for boreal summer and fall conditions, and the spatial structure of variability is more consistent with observed meridional mode variability.

The role of hemispheric asymmetry in optimal initial conditions that produce transient growth was examined through developing a Northern or Southern Hemisphere initial norm under which to evaluate growth. Leading optimal conditions grow more when they start in the Northern Hemisphere compared to the Southern Hemisphere during boreal spring, and SH-constrained initial conditions do not grow at all during boreal fall. This is consistent with existing research that finds an important role for Northern Hemisphere compared to Southern Hemisphere extratropical forcing in meridional mode variability. Although we caution that these optimals were calculated by assuming that SST influences the atmosphere through its impact on deep convection. A different result may result in a model where SST is coupled to the atmosphere through boundary layer SST-induced pressure variations, an effect that we have not considered in depth in this work.

Optimal structures and growth were examined for different mean states, with a focus on ITCZ width and 
location as the WES feedback is relatively insensitive to seasonal variations. For an equatorially symmetric ITCZ optimal structures were either purely symmetric or antisymmetric about the equator and evolve conserving their initial symmetry. Internal dynamics do not mix the symmetries for this idealized case. This does not preclude external shocks (like asymmetric external atmospheric forcing) from mixing the symmetry of the optimals. For narrow ITCZ structures equatorially symmetric optimals dominate the growth, while antisymmetric optimal structures dominate growth for wider ITCZ structures. This is traced to the increase in importance of the antisymmetric atmospheric Rossby wave response as the (symmetric) effective coupling region gets broader.

Growth and optimal structures were also examined as a function of ITCZ location, which relates to seasonal variations in the ITCZ. In that case, the optimal response activity was mainly contained in the same hemisphere as the ITCZ, as there is not enough convective support for an atmospheric response that would feed back and grow outside of the ITCZ region. As a consequence of this, optimals under an asymmetric ITCZ are neither symmetric nor antisymmetric. Interestingly, optimal initial conditions experience maximum growth when the ITCZ is located off of the equator, which is counterintuitive considering that maximum meridional mode variance is found in boreal spring (Chiang and Vimont 2004). This could imply that meridional mode variability during boreal summer and fall is more transiently maintained by internal ocean-atmosphere interactions, whereas in spring the thermodynamic coupling might have a secondary role to direct atmospheric forcing. More studies are needed to assess the relative roles of internal thermodynamically coupled ocean-atmosphere interactions and direct atmospheric forcing on meridional mode variability during different seasons.

There are numerous caveats to the findings in this study. First, the atmospheric model is overly simplistic, although the results should be applicable to a Lindzen and Nigam (1987) or Battisti et al. (1999) style model of the boundary layer responding to surface temperature anomalies. Second, the ocean model is clearly too simplistic as lateral Ekman fluxes, upwelling, ocean dynamics, and low-cloud feedbacks will play a role in surface temperature variations in nature. Third, in reality the ITCZ and WES feedback are codetermined by the dynamics of the Hadley and Walker circulations. No attempt was made herein to ensure a physically consistent mean state, aside from deriving the specific mean states for the Atlantic Ocean from observations.

Despite these caveats, the study does provide physical insight into meridional mode behavior under structural variations in the mean state. Specifically, it highlights the importance of the mean state as a "mode selector" of thermodynamically coupled ocean-atmosphere variability; that is, through preferentially enabling oceanatmosphere coupling for specific atmospheric modes, the mean state can play an important role in determining whether tropical thermodynamically coupled variability is dominated by equatorially symmetric, antisymmetric, or asymmetric structures. Results also identify the convective coupling provided by the ITCZ as a key player in meridional mode dynamics.

Acknowledgments. This work was supported by NSF Climate and Large Scale Dynamics Projects ATM0849689 and 1463970 and the University of Wisconsin Climate, People, and the Environment Program. We thank Tim Li for handling the manuscript; and S.-P. Xie, A. Subramanian, and one anonymous reviewer for their comments and suggestions.

\section{APPENDIX A}

\section{$K_{q}(y) \alpha(y)$ as the Effective Coupling of the System}

Let us reorder the state vector as $\boldsymbol{\Phi}=(T, u, v, \phi)^{\mathrm{T}}$. In this way the matrix $\mathbf{M}$ for an individual point $y_{i}$ is the following:

$$
\mathbf{M}=\left[\begin{array}{cccc}
-\varepsilon_{T}+\gamma \nabla^{2} & \alpha(y) & 0 & 0 \\
0 & -\varepsilon & y & -i k \\
0 & -y & -\varepsilon & -\frac{\partial}{\partial y} \\
K_{q}(y) & -i k & -\frac{\partial}{\partial y} & -\varepsilon
\end{array}\right] .
$$

The $\partial / \partial y$ is a numerical derivative, and connects this block to similar blocks acting on $y_{i-1}$ and $y_{i+1}$.

To find the spectra $\left\{\Phi_{j},-i \omega_{j}\right\}$ of $\mathbf{M}$ we can solve the determinant $\left|\mathbf{M}+i \omega_{j} \mathbf{1}\right|=0$ :

$$
\left|\begin{array}{cccc}
-\varepsilon_{T}+\gamma \nabla^{2}+i \omega_{j} & \alpha(y) & 0 & 0 \\
0 & -\varepsilon+i \omega_{j} & y & -i k \\
0 & -y & -\varepsilon+i \omega_{j} & -\frac{\partial}{\partial y} \\
K_{q}(y) & -i k & -\frac{\partial}{\partial y} & -\varepsilon+i \omega_{j}
\end{array}\right|=0 .
$$

We can separate this in an uncoupled part (Matsuno atmospheric model; Matsuno 1966) and a part that contains the coupling: 


$$
\left(-\varepsilon_{T}+\gamma \nabla^{2}+i \omega_{j}\right)\left|\begin{array}{ccc}
-\varepsilon+i \omega_{j} & y & -i k \\
-y & -\varepsilon+i \omega_{j} & -\frac{\partial}{\partial y} \\
-i k & -\frac{\partial}{\partial y} & -\varepsilon+i \omega_{j}
\end{array}\right|-\alpha\left|\begin{array}{ccc}
0 & y & -i k \\
0 & -\varepsilon+i \omega_{j} & -\frac{\partial}{\partial y} \\
-K_{q} & -\frac{\partial}{\partial y} & -\varepsilon+i \omega_{j}
\end{array}\right|=0 .
$$

We can further decompose the last determinant as follows:

$$
\left(-\varepsilon_{T}+\gamma \nabla^{2}+i \omega_{j}\right)\left|\begin{array}{ccc}
-\varepsilon+i \omega_{j} & y & -i k \\
-y & -\varepsilon+i \omega_{j} & -\frac{\partial}{\partial y} \\
-i k & -\frac{\partial}{\partial y} & -\varepsilon+i \omega_{j}
\end{array}\right|+K_{q} \alpha\left|\begin{array}{cc}
y & -i k \\
-\varepsilon+i \omega_{j} & -\frac{\partial}{\partial y}
\end{array}\right|=0 .
$$

This shows that the coupled part of the system depends on the product $K_{q}(y) \alpha(y)$. If the system were uncoupled $\left[K_{q}(y) \alpha(y)=0\right]$, the eigenvalues would just be the ones given by the Matsuno atmospheric model (first determinant) plus another one equal to the oceanic damping. We further notice that the Matsuno model is normal, so the source of nonnormality comes from the effective coupling.

\section{APPENDIX B}

\section{Relation between Leading Constrained and Unconstrained Optimals}

Any optimal can be decomposed into an equatorially symmetric part $0.5 \mathbf{p}_{\text {sym }}$ and an antisymmetric part $0.5 \mathbf{p}_{\text {ant }}$. As a consequence we can write an optimal constrained to start in the $\mathrm{NH} \mathbf{p}_{\mathrm{NH}}$, for example, as follows:

$$
\mathbf{p}_{\mathrm{NH}}=\frac{1}{2} \mathbf{p}_{\mathrm{sym}}+\frac{1}{2} \mathbf{p}_{\mathrm{ant}},
$$

where $\mathbf{p}_{\text {sym }}$ has the same amplitude as $\mathbf{p}_{\text {ant }}\left(\right.$ and $\left.\mathbf{p}_{\mathrm{NH}}\right)$ in the $\mathrm{NH}$.

For a symmetric mean state both $\mathbf{p}_{\text {sym }}$ and $\mathbf{p}_{\text {ant }}$ will evolve conserving their symmetry. In this case, applying $\mathbf{G}_{\tau}^{\dagger} \mathbf{G}_{\tau}$ [see (8)] to (B1), we find that either $\mathbf{p}_{\text {sym }}$ or $\mathbf{p}_{\text {ant }}$ is equal to the leading unconstrained optimal $\mathbf{p}$. In this way we can write

$$
\mathbf{p}_{\mathrm{NH}}=\frac{1}{2} \mathbf{p}+\frac{1}{2} \hat{\mathbf{p}},
$$

where $\hat{\mathbf{p}}$ is the opposite symmetry structure such that it cancels the contribution of $\mathbf{p}$ in the SH. This implies that the constrained optimal will just be "half" the unconstrained optimal.
For example, in this case Fig. 3c corresponds to $\mathbf{p}_{\mathrm{NH}}$, the unconstrained optimal $\mathbf{p}$ corresponds to $\mathbf{p}_{\text {ant }}$ (Fig. 3a), and $\hat{\mathbf{p}}=\mathbf{p}_{\text {sym }}$. A similar idea holds (approximately) for Fig. 4, but in that case $\mathbf{p}=\mathbf{p}_{\text {sym }}$ and $\hat{\mathbf{p}}=\mathbf{p}_{\text {ant }}$.

\section{REFERENCES}

Battisti, D. S., E. S. Sarachik, and A. C. Hirst, 1999: A consistent model for the large-scale steady surface atmospheric circulation in the tropics. J. Climate, 12, 2956-2964, doi:10.1175/ 1520-0442(1999)012<2956:ACMFTL>2.0.CO;2.

Bischoff, T., and T. Schneider, 2014: Energetic constraints on the position of the intertropical convergence zone. J. Climate, 27, 4937-4951, doi:10.1175/JCLI-D-13-00650.1.

Chang, P., L. Ji, and H. Li, 1997: A decadal climate variation in the tropical Atlantic Ocean from thermodynamic air-sea interactions. Nature, 385, 516-518, doi:10.1038/385516a0.

_ , L. Zhang, R. Saravanan, D. J. Vimont, J. C. H. Chiang, L. Ji, H. Seidel, and M. K. Tippett, 2007: Pacific meridional mode and El Niño-Southern Oscillation. Geophys. Res. Lett., 34, L16608, doi:10.1029/2007GL030302.

Chiang, J. C. H., and D. J. Vimont, 2004: Analogous Pacific and Atlantic meridional modes of tropical atmosphere-ocean variability. J. Climate, 17, 4143-4158, doi:10.1175/JCLI4953.1. , S. E. Zebiak, and M. A. Cane, 2001: Relative roles of elevated heating and surface temperature gradients in driving anomalous surface winds over tropical oceans. J. Atmos. Sci., 58, 1371-1394, doi:10.1175/1520-0469(2001)058<1371: RROEHA $>2.0 . \mathrm{CO} ; 2$.

Clement, A., P. DiNezio, and C. Deser, 2011: Rethinking the ocean's role in the Southern Oscillation. J. Climate, 24, 40564072, doi:10.1175/2011JCLI3973.1.

Czaja, A., 2004: Why is north tropical Atlantic SST variability stronger in boreal spring? J. Climate, 17, 3017-3025, doi:10.1175/1520-0442(2004)017<3017:WINTAS>2.0.CO;2.

, P. van der Vaart, and J. Marshall, 2002: A diagnostic study of the role of remote forcing in tropical Atlantic variability. J. Climate, 15, 3280-3290, doi:10.1175/1520-0442(2002)015<3280: ADSOTR $>2.0 . \mathrm{CO} ; 2$.

Dee, D. P., and Coauthors, 2011: The ERA-Interim reanalysis: Configuration and performance of the data assimilation system. Quart. J. Roy. Meteor. Soc., 137, 553-597, doi:10.1002/qj.828. 
Enfield, D. B., A. M. Mestas-Nuñez, D. A. Mayer, and L. CidSerrano, 1999: How ubiquitous is the dipole relationship in tropical Atlantic sea surface temperatures? J. Geophys. Res., 104, 7841, doi:10.1029/1998JC900109.

Evan, A. T., R. J. Allen, R. Bennartz, and D. J. Vimont, 2013: The modification of sea surface temperature anomaly linear damping time scales by stratocumulus clouds. J. Climate, 26, 3619-3630, doi:10.1175/JCLI-D-12-00370.1.

Farrell, B. F., and P. J. Ioannou, 1996: Generalized stability theory. Part I: Autonomous operators. J. Atmos. Sci., 53, 2025-2040, doi:10.1175/1520-0469(1996)053<2025:GSTPIA > 2.0.CO;2.

Frierson, D. M. W., and Coauthors, 2013: Contribution of ocean overturning circulation to tropical rainfall peak in the Northern Hemisphere. Nat. Geosci., 6, 940-944, doi:10.1038/ ngeo1987.

Gill, A. E., 1980: Some simple solutions for heat-induced tropical circulation. Quart. J. Roy. Meteor. Soc., 106, 447-462, doi:10.1002/qj.49710644905.

Hastenrath, S., and L. Heller, 1977: Dynamics of climatic hazards in Northeast Brazil. Quart. J. Roy. Meteor. Soc., 103, 77-92, doi:10.1002/qj.49710343505.

Hirst, A. C., 1986: Unstable and damped equatorial modes in simple coupled ocean-atmosphere models. J. Atmos. Sci., 43, 606-632, doi:10.1175/1520-0469(1986)043<0606: UADEMI $>2.0 . \mathrm{CO} ; 2$.

Houghton, R. W., and Y. M. Tourre, 1992: Characteristics of low-frequency sea surface temperature fluctuations in the tropical Atlantic. J. Climate, 5, 765-772, doi:10.1175/ 1520-0442(1992)005<0765:COLFSS >2.0.CO;2.

Kossin, J. P., and D. J. Vimont, 2007: A more general framework for understanding Atlantic hurricane variability and trends. Bull. Amer. Meteor. Soc., 88, 1767-1781, doi:10.1175/ BAMS-88-11-1767.

Lindzen, R. S., and S. Nigam, 1987: On the role of sea surface temperature gradients in forcing low-level winds and convergence in the tropics. J. Atmos. Sci., 44, 2418-2436, doi:10.1175/ 1520-0469(1987)044<2418:OTROSS > 2.0.CO;2.

Linkin, M. E., and S. Nigam, 2008: The North Pacific Oscillationwest Pacific teleconnection pattern: Mature-phase structure and winter impacts. J. Climate, 21, 1979-1997, doi:10.1175/ 2007JCLI2048.1.

Matsuno, T., 1966: Quasi-geostrophic motions in the equatorial area. J. Meteor. Soc. Japan, 44, 25-43.

Moura, A. D., and J. Shukla, 1981: On the dynamics of droughts in Northeast Brazil: Observations, theory, and numerical experiments with a general circulation model. J. Atmos. Sci., 38, 2653-2675, doi:10.1175/1520-0469(1981)038<2653: OTDODI $>2.0 . \mathrm{CO} ; 2$.

Neelin, J. D., 1989: On the interpretation of the Gill model. J. Atmos. Sci., 46, 2466-2468, doi:10.1175/1520-0469(1989)046<2466: OTIOTG $>2.0 . \mathrm{CO} ; 2$.

Nobre, P., and J. Shukla, 1996: Variations of sea surface temperature, wind stress, and rainfall over the tropical Atlantic and South America. J. Climate, 9, 2464-2479, doi:10.1175/ 1520-0442(1996)009<2464:VOSSTW>2.0.CO;2.

Noguchi, H., 1998: Coupled ocean-atmospheric waves due to windevaporation-SST feedback (in Japanese). M.S. thesis, Graduate School of Environmental Science, Hokkaido University, $56 \mathrm{pp}$.

Okajima, H., S.-P. Xie, and A. Numaguti, 2003: Interhemispheric coherence of tropical climate variability: Effect of the climatological ITCZ. J. Meteor. Soc. Japan, 81, 1371-1386, doi:10.2151/ jmsj.81.1371.
Penland, C., and L. M. Hartten, 2014: Stochastic forcing of north tropical Atlantic sea surface temperatures by the North Atlantic Oscillation. Geophys. Res. Lett., 41, 2126-2132, doi:10.1002/2014GL059252.

Rogers, J. C., 1981: The North Pacific Oscillation. J. Climatol., 1, 39-57, doi:10.1002/joc.3370010106.

Schneider, T., T. Bischoff, and G. H. Haug, 2014: Migrations and dynamics of the intertropical convergence zone. Nature, $\mathbf{5 1 3}$ 45-53, doi:10.1038/nature13636.

Servain, J., I. Wainer, J. P. McCreary, and A. Dessier, 1999: Relationship between the equatorial and meridional modes of climatic variability in the tropical Atlantic. Geophys. Res. Lett., 26, 485-488, doi:10.1029/1999GL900014.

Smirnov, D., and D. J. Vimont, 2011: Variability of the Atlantic meridional mode during the Atlantic hurricane season. J. Climate, 24, 1409-1424, doi:10.1175/2010JCLI3549.1.

Takahashi, K., and D. S. Battisti, 2007: Processes controlling the mean tropical Pacific precipitation pattern. Part I: The Andes and the eastern Pacific ITCZ. J. Climate, 20, 3434-3451, doi:10.1175/JCLI4198.1.

Tanimoto, Y., and S.-P. Xie, 2002: Inter-hemispheric decadal variations in SST, surface wind, heat flux and cloud cover over the Atlantic Ocean. J. Meteor. Soc. Japan, 80, 1199-1219, doi:10.2151/jmsj.80.1199.

Toma, V. E., and P. J. Webster, 2010a: Oscillations of the intertropical convergence zone and the genesis of easterly waves. Part I: Diagnostics and theory. Climate Dyn., 34, 587604, doi:10.1007/s00382-009-0584-x.

$\longrightarrow$, and 2010b: Oscillations of the intertropical convergence zone and the genesis of easterly waves. Part II: Numerical verification. Climate Dyn., 34, 605-613, doi:10.1007/ s00382-009-0585-9.

Tomas, R. A., and P. J. Webster, 1997: The role of inertial instability in determining the location and strength of nearequatorial convection. Quart. J. Roy. Meteor. Soc., 123, 14451482, doi:10.1002/qj.49712354202.

Vimont, D. J., 2010: Transient growth of thermodynamically coupled variations in the tropics under an equatorially symmetric mean state. J. Climate, 23, 5771-5789, doi:10.1175/ 2010JCLI3532.1.

_ M. Alexander, and A. Fontaine, 2009: Midlatitude excitation of tropical variability in the Pacific: The role of thermodynamic coupling and seasonality. J. Climate, 22, 518-534, doi:10.1175/2008JCLI2220.1.

Wang, S. Y., M. L'Heureux, and H. H. Chia, 2012: ENSO prediction one year in advance using western North Pacific sea surface temperatures. Geophys. Res. Lett., 39, L05702, doi:10.1029/2012GL050909.

Wu, R., B. P. Kirtman, and V. Krishnamurthy, 2008: An asymmetric mode of tropical Indian Ocean rainfall variability in boreal spring. J. Geophys. Res., 113, D05104, doi:10.1029/ 2007JD009316.

Xie, S.-P., 1996: Westward propagation of latitudinal asymmetry in a coupled ocean-atmosphere model. J. Atmos. Sci., 53, 3236-3250, doi:10.1175/1520-0469(1996)053<3236: WPOLAI $>2.0 . \mathrm{CO} ; 2$. , 1999: A dynamic ocean-atmosphere model of the tropical Atlantic decadal variability. J. Climate, 12, 64-70, doi:10.1175/ 1520-0442-12.1.64.

, and S. G. H. Philander, 1994: A coupled oceanatmosphere model of relevance to the ITCZ in the eastern Pacific. Tellus, 46A, 340-350, doi:10.1034/ j.1600-0870.1994.t01-1-00001.x. 
- and Y. Tanimoto, 1998: A pan-Atlantic decadal climate oscillation. Geophys. Res. Lett., 25, 2185-2188, doi:10.1029/98GL01525. , and K. Saito, 2001: Formation and variability of a northerly ITCZ in a hybrid coupled AGCM: Continental forcing and oceanic-atmospheric feedback. J. Climate, 14, 1262-1276, doi:10.1175/1520-0442(2001)014<1262:FAVOAN > 2.0.CO;2.

- Y. Tanimoto, H. Noguchi, and T. Matsuno, 1999: How and why climate variability differs between the tropical Atlantic and Pacific. Geophys. Res. Lett., 26, 1609-1612, doi:10.1029/ 1999GL900308.
Zebiak, S. E., 1986: Atmospheric convergence feedback in a simple model for El Niño. Mon. Wea. Rev., 114, 1263-1271, doi:10.1175/1520-0493(1986)114<1263:ACFIAS > 2.0.CO;2.

Zhang, H., A. Clement, and P. D. Nezio, 2014a: The South Pacific meridional mode: A mechanism for ENSO-like variability. J. Climate, 27, 769-783, doi:10.1175/JCLI-D-13-00082.1.

, C. Deser, A. Clement, and R. A. Tomas, 2014b: Equatorial signatures of the Pacific meridional modes: Dependence on mean climate state. Geophys. Res. Lett., 41, 568-574, doi:10.1002/ 2013 GL058842. 